\title{
The value of nasopharyngeal aspirate, gastric aspirate and bronchoalveolar lavage fluid in the diagnosis of childhood tuberculosis
}

\author{
Erkan Çakır ${ }^{1}$, Ali Özdemir ${ }^{2}$, Hayrettin Daşkaya ${ }^{3}$, Tarık Umutoğlu ${ }^{3}$, Mine Yüksel ${ }^{4}$ \\ Departments of ${ }^{1}$ Pediatric Pulmonology, ${ }^{3}$ Anesthesiology and ${ }^{4}$ Pediatrics, Bezmialem Vakif University, Istanbul; Department \\ of Pediatrics ${ }^{2}$ Pediatric Pulmonology, Mersin City Hospital, Mersin, Turkey. \\ E-mail: aliozdemir@hotmail.com
}

Received: 7 June 2017, Revised: 18th July 2017, Accepted: 16th August 2017

\begin{abstract}
SUMMARY: Çakır E, Özdemir A, Daşkaya H, Umutoğlu T, Yüksel M. The value of nasopharyngeal aspirate, gastric aspirate and bronchoalveolar lavage fluid in the diagnosis of childhood tuberculosis. Turk J Pediatr 2018; 60: 10-13.

Pulmonary tuberculosis (TB) is an important cause of morbidity and mortality especially in developing countries. A definitive microbiologic confirmation of Mycobacterium tuberculosis is important in the diagnosis of childhood TB. We aimed to compare the diagnostic value of nasopharyngeal aspirate (NPA), gastric aspirate (GA) and bronchoalveolar lavage (BAL) specimens in children with highly suspected pulmonary tuberculosis (TB). NPA, GA and BAL samples were obtained from forty patients. The mean age was $9.2 \pm 4.7$ years. Sixtyeight percent of children had a history of household contact and $82 \%$ had tuberculin skin test positivity. Acid-fast bacilli (AFB) stain was positive in $22.5 \%(\mathrm{~N}=9)$ of BAL, $17.5 \%(\mathrm{~N}=7)$ of $\mathrm{GA}$, and $10 \%(\mathrm{~N}=4)$ of NPA samples. Positive Lowenstein-Jensen culture was $27.5 \%(\mathrm{~N}=11)$ in BAL, 22.5\% $(\mathrm{N}=9)$ in $\mathrm{GA}$, and $12.5 \%(\mathrm{~N}=5)$ in NPA samples. Positive AFB stains and growth in TB cultures from BAL fluid and GA samples were both higher than NPA samples $(p<0.006$ and $p<0.004$, respectively GA). We conclude that NPA specimen fails to determine Mycobacterium tuberculosis in children with highly suspected pulmonary TB when compared to GA or BAL fluid.
\end{abstract}

Key words: nasopharyngeal aspirate, gastric aspirate, bronchoalveolar lavage fluid, childhood pulmonary tuberculosis, Mycobacterium tuberculosis, diagnosis.

Tuberculosis (TB) is an important cause of morbidity and mortality especially in developing countries. Children comprise an estimated 10$20 \%$ of all TB cases in TB-endemic communities. The diagnosis of pulmonary TB is challenging in children because symptoms are nonspecific, sputum or smear microscopy positivity is less than $10-15 \%$ of all cases, and cultures yield low positive results $(20-40 \%)$. This is mainly due to the paucibacillary characteristic of the childhood TB. ${ }^{1-3}$

The diagnostic criteria of childhood pulmonary $\mathrm{TB}$, which includes respiratory symptoms, known adult contact with active TB, a positive tuberculin skin test (TST) and suggestive signs on the chest radiograph, have not changed for many years. A definitive microbiologic confirmation of Mycobacterium tuberculosis is still important in the diagnosis of childhood TB. Common recommendation for microbiological investigation in children is based on three consecutive early morning fasting gastric aspirates (GA) in the lack of sputum production. ${ }^{4}$ Additionally, studies suggest that combining GA and bronchoalveolar lavage (BAL) fluid may improve bacteriological yield in children with pulmonary TB. ${ }^{3,5,6}$ However, GA collection usually requires hospital admission and BAL, performed by flexible bronchoscopy, is a highly specialized procedure. There are only a few studies that suggest nasopharyngeal aspirates (NPA) might be compatible with previously mentioned methods in determining $\mathrm{TB}$ in children. This method offers an advantage with no requirement of hospital admission and can be easily performed at any time of the day. ${ }^{7-9}$ 
In this study, we aim to compare the diagnostic value of NPA, GA and BAL fluid specimens in children with highly suspected pulmonary $\mathrm{TB}$.

\section{Material and Methods}

The study was conducted at the Pediatric Pulmonology Section of the Bezmialem Vakif University. The study was approved by the Bezmialem Vakif University local ethical committee. Written informed consent was obtained from parents.

\section{Pulmonary tuberculosis (TB) patients}

Children with a strong suspicion of pulmonary TB in whom fiberoptic flexible bronchoscopy (FB) performed were enrolled to the study. In our institution $\mathrm{FB}$ is a routine procedure for suspected TB patients with clinical or radiological abnormalities such as brassy cough, localized wheezing, large lymphadenopathy, hemoptysis, presence of consolidation or atelectasis, and hyperinflation. FB is also performed on patients who have persistent clinical symptoms or radiological abnormalities despite appropriate TB treatment.

Our study consisted of forty children who underwent flexible bronchoscopy for highly suspected pulmonary TB. The diagnosis of TB disease was established in accordance with the World Health Organization's TB Standards case definition. ${ }^{1}$ This was based on respiratory symptoms, radiological findings, tuberculin skin test positivity, history of contact with active $\mathrm{TB}$, and acid fast bacillus and MTB culture positivity. Tuberculin skin test results were interpreted according to the recommendations of the American Thoracic Society and the Centers for Disease Control. ${ }^{10}$ Among patients, $68 \%$ of children had a history of household contact and $82 \%$ had tuberculin skin test positivity. Their most frequent symptoms were cough $(80 \%)$, weakness and anorexia (50\%), weight loss (45\%), and night sweats $(42.5 \%)$, respectively. All the patients had consistent radiological findings unresponsive to non-specific antibiotherapy. Eighty-five percent $(n=34)$ of the patients had primary TB disease, and $15 \%(n=6)$ had adult-type secondary TB disease. All patients responded to the antituberculosis therapy.

Nasopharyngeal aspirates (NPA), gastric aspirates (GA) and bronchoalveolar lavage
(BAL) fluid were obtained from all patients.

\section{Gastric aspiration samples}

All patients were admitted to the hospital for early morning fasting GA samples. Nasogastric tubes (minimum 10 French size) were used to obtain stomach contents on three consecutive days into a sterile syringe. If less than $10 \mathrm{ml}$ of sample was aspirated, we instilled $20-30 \mathrm{ml}$ of sterile water and quickly withdrew into the tube. Then, the specimen was transported to the laboratory immediately.

\section{Nasopharyngeal aspiration samples}

Patients were in upright position. A suction catheter was inserted through the nostril into the oropharynx which stimulated a cough reflex; then $5 \mathrm{ml}$ of isotonic saline was injected and subsequently aspirated into a sterile syringe.

\section{$B A L$ samples with flexible bronchoscopy}

All FBs were performed on TB patients in the procedure room using Pentax EB-1570K (4.9 $\mathrm{mm}$, Hoya Corporation, Tokyo, Japan) Pentax EB-1170K (3.7 mm, Hoya Corporation, Tokyo, Japan), Fujinon FB-120P (2.8 mm, Fujifilm Corporation, Tokyo, Japan). FB was performed either with a laryngeal airway mask or transnasally. During the procedure, all patients were monitored for oxygen saturation and cardiac rhythm. Patients received midazolam, pethidine hydrochloride (meperidine), ketamine or propofol as premedication and lidocaine was used as a topical anesthetic, applied locally with a maximum dose of $4 \mathrm{mg} / \mathrm{kg}$. Bronchoalveolar lavage samples were obtained either from the area of radiological abnormality or right middle lobe/left lingular lobe in the absence of localization by wedging the tip of bronchoscopy into a subsegmental bronchus; $1 \mathrm{ml} / \mathrm{kg}$ (maximum $20 \mathrm{ml}$ ) isotonic saline solution was instilled via the bronchoscopy which was followed by immediate suction of the fluid into a sterile container. There was no major complication observed during the procedure. Two patients had transient hypoxia which was resolved by nasal oxygen.

The samples (NPA, GA and BAL fluid) collected from patients were immediately processed for screening acid-fast bacilli (AFB) with rhodamine-auramine and Erlich Ziehl-Nielsen staining which was followed by culturing in Lowenstein-Jensen medium. 
Table I. Microbiologic Results of the Specimens According to the Method Used.

\begin{tabular}{|c|c|c|c|c|c|}
\hline \multirow[b]{2}{*}{ Examination } & \multicolumn{3}{|c|}{ Methods } & \multicolumn{2}{|c|}{$\mathrm{P}$ value } \\
\hline & $\begin{array}{l}\text { Nasopharyngeal } \\
\text { aspirate (NPA) }\end{array}$ & $\begin{array}{l}\text { Gastric aspirate } \\
\text { (GA) }\end{array}$ & $\begin{array}{l}\text { Bronchoalveolar } \\
\text { lavage (BAL) }\end{array}$ & GA versus NPA & BAL versus NPA \\
\hline $\begin{array}{l}\text { Acid-fast bacilli, } \\
\text { N (\%) }\end{array}$ & $4(10.0)$ & $7(17.5)$ & $9(22.5)$ & 0.003 & 0.005 \\
\hline $\begin{array}{l}\text { Positive culture, } \\
\mathrm{N}(\%)\end{array}$ & $5(12.5)$ & $9(22.5)$ & $11(27.5)$ & 0.004 & 0.006 \\
\hline
\end{tabular}

\section{Statistical analysis}

Statistical analysis was performed using IBM SPSS-19 program. Mean, median, standard deviation and interquartile were used for metric variables. Categorical data was evaluated using the Chi-square test and a $\mathrm{p}$ value $<0.05$ was accepted as statistically significant.

\section{Results}

Forty children (55\% female) were enrolled to the study. The patients' age ranged from 1 to 16 years (mean $9.2 \pm 4.7$ years). AFB stains were positive in $22.5 \%(\mathrm{n}=9)$ of BAL fluid, $17.5 \%(n=7)$ of GA, and $10 \%(n=4)$ of NPA samples. Lowenstein-Jensen culture positive result was $27.5 \%(\mathrm{n}=11)$ in BAL fluid, $22.5 \%(n=9)$ in $\mathrm{GA}$, and $12.5 \%(\mathrm{n}=5)$ in NPA samples. (Table 1 ). The age range for GA, BAL and NPA culture positive patients were 1-15 years, 1-16 years and 3-12 years, respectively $(\mathrm{p}>0.05)$. All AFB stain positive patients [BAL group 9/11 (81.8\%), GA group 7/9 (77.8\%), and NPA group 4/5 $(80.0 \%)$, respectively)] had grown Mycobacterium tuberculosis in their cultures. Positive AFB stains and growth in TB cultures from GA samples and BAL fluid were both higher than NPA samples (GA versus NPA, $\mathrm{p}<0.003$ and BAL versus NFA, $\mathrm{p}<0.005$ for AFB stains; and GA versus NPA, $\mathrm{p}<0.004$ and BAL versus NPA, $\mathrm{p}<0.006$ for TB cultures). However, there were no difference in positive AFB microscopy and TB isolation from cultures between BAL fluid and GA samples ( $p>0.05)$. Notably, no drug resistance was identified in cultures. All patients responded to standard anti-tuberculosis treatment.

\section{Discussion}

The bacteriological diagnosis of pulmonary TB presents challenges in children. Sputum is usually non-reachable and various methods of collecting specimens have limitations in isolating Mycobacterium tuberculosis due to low bacterial yield. The most common method in the absence of sputum is three consecutive day GA collection which usually requires hospital admission. Another method of detecting Mycobacterium tuberculosis is BAL fluid by using a FB which may not be achievable in every setting. These methods give an approximately $20-30 \%$ of Mycobacterium tuberculosis identification in cultures in the favor of BAL fluid. Additionally, when both methods are used together, the positive culture result may achieve as high as $40 \% .^{1-5}$

Lately, some studies have shown that Mycobacterium tuberculosis could be determined in NPA samples in children with pulmonary TB. ${ }^{7-9}$ This procedure offers an advantage in performing and simpler than other previously mentioned methods. There are few studies in different settings that investigated NPA specimens with other various samples [culture, polymerase chain reaction (PCR) testing, GA, induced sputum (IS) and stool] with conflicting results in pulmonary $\mathrm{TB}$ children. Owens et al. ${ }^{7}$ compared NPA and hypertonic saline IS samples in suspected pulmonary TB children. They reported smear positivity $8.5 \%$ and culture positivity $23.9 \%$ in NPA versus smear positivity $9.6 \%$ and culture positivity $21.6 \%$ in IS samples. Thus, they concluded that NPA was compatible with IS in the identification of Mycobacterium tuberculosis. Later, Al-Aghbari et al. ${ }^{8}$ studied one NPA, three GA and three expectorated/induced sputa over 3 consecutive days in children with pulmonary TB. ${ }^{8}$ They diagnosed $29(13.6 \%)$ children by culture and $18(8.5 \%)$ by smear microscopy. Both culture and smear positivity did not differ from each other in 3 different methods. Furthermore, subsequent day of culturing the samples did not result in additional gain. In contrast to these 
studies, a recent report from Oberhelman et al. ${ }^{9}$ observed that $\mathrm{GA}(\mathrm{N}=22)$ was superior to NPA $(\mathrm{N}=12)$ and stool $(\mathrm{N}=4)$ specimens in 38 Mycobacterium tuberculosis recovered cultures.

There are also some differently designed studies investigating alternative specimen methods for TB diagnosis in children. Zar et al. ${ }^{11}$ compared 535 children with suspected pulmonary TB (21.9\% were HIV-infected) IS and NPA specimens using smear, liquid culture and Xpert MTB/RF methods. A positive smear, Xpert test or culture occurred in 30 (5.6\%), 81 $(15.1 \%)$ and 87 children $(16.3 \%)$, respectively. The observed culture yield was higher in IS (96.6\%) than NPA (70.1\%), similar for IS $(71 \%)$ and NPA $(65 \%)$ by Xpert tests, and smear positivity was lower for both IS (33\%) and NPA $(25 \%)$ in their study. In a recent study examining alternative specimens (GA, sputum, NPA and stool) for TB diagnosis in HIV-infected children using X-pert MTB/RF method, the combination of NFA and stool sample showed an alternative promising and rapid confirmation of childhood $\mathrm{TB}$ with a sensitivity of $75 \% .{ }^{12}$

In our study, Mycobacterium tuberculosis was isolated in approximately $25 \%$ of cultures in cases with suspected pulmonary TB both in BAL fluid and GA but less in NPA samples (12.5\%). We observed that in NPA specimen group, both culture and smear was less diagnostic in identifying Mycobacterium tuberculosis than BAL fluid and GA. To our knowledge, there are no studies that compare these three methods in children with pulmonary TB in the literature.

In conclusion, our data suggests that NPA specimen fails to determine Mycobacterium tuberculosis in children with highly suspected pulmonary TB when compared to GA and BAL fluid. The major limitation of our study is limited number of children with pulmonary tuberculosis. Therefore, further studies with compatible patient population are needed in order to clarify the validity of NPA in the diagnosis of pulmonary TB in children.

\section{REFERENCES}

1. Stop TB Partnership Childhood TB Subgroup, World Health Organization Guidance for National Tuberculosis Programmes on the management of tuberculosis in children. Chapter 1: Introduction and diagnosis of tuberculosis in children. Int J Tuberc Lung Dis 2006; 10: 1091-1097.

2. Marais BJ, Pai M. Specimen collection methods in the diagnosis of childhood tuberculosis. Indian J Med Microbiol 2006; 24: 249-251.

3. Cakir E, Kut A, Ozkaya E, Gedik AH, Midyat L, Nursoy M. Bronchoscopic evaluation in childhood pulmonary tuberculosis: risk factors of airway involvement and contribution to the bacteriologic diagnosis. Pediatr Infect Dis J 2013; 32: 921-923.

4. Pomputius WF III, Rost J, Dennehy PH, Carter EJ. Standardization of gastric aspirate technique improves yield in the diagnosis of tuberculosis in children. Pediatr Infect Dis J 1997; 16: 222-226.

5. Singh M, Moosa NV, Kumar L, Sharma M. Role of gastric lavage and broncho-alveolar lavage in the bacteriological diagnosis of childhood pulmonary tuberculosis. Indian Pediatr 2000; 37: 947-951.

6. Somu N, Swaminathan S, Paramasivan $\mathrm{CN}$, et al Value of bronchoalveolar lavage and gastric lavage in the diagnosis of pulmonary tuberculosis in children. Tuber Lung Dis 1995; 76: 295-299.

7. Owens S, Abdel-Rahman IE, Balyejusa S, et al. Nasopharyngeal aspiration for diagnosis of pulmonary tuberculosis. Arch Dis Child 2007; 92: 693-696.

8. Al-Aghbari N, Al-Sonboli N, Yassin MA, et al. Multiple sampling in one day to optimize smear microscopy in children with tuberculosis in Yemen. Plos One 4 2009: e5140.

9. Oberhelman RA, Soto-Castellares G, Gilman RH, et al. Diagnostic approaches for paediatric tuberculosis by use of different specimen types, culture methods, and PCR: A prospective case-control study. Lancet Infect Dis 2010; 10: 612-620.

10. Bass JB. Tuberculin test, preventive therapy, and elimination of tuberculosis. Am Rev Respir Dis 1990;141: 812-813.

11. Zar HJ, Workman L, Isaacs W, et al. Rapid molecular diagnosis of pulmonary tuberculosis in children using nasopharyngeal specimens. Clin Infect Dis 2012; 55: 1088-1095.

12. Marcy O, Ung V, Goyet S, et al. Performance of Xpert MTB/RIF and alternative specimen collection methods for the diagnosis of tuberculosis in HIV-infected children. Clin Infect Dis 2016; 62: 1161-1168. 